\title{
Condiciones actuales en términos de pérdida de biodiversidad en corredores biológicos de la Granja Agroecológica Uniminuto, Villavicencio, Meta, Colombia
}

\section{Current conditions in terms of loss of biodiversity in biological corridors of the Uniminuto Agro- ecological Farm, Villavicencio, Meta, Colombia}

\author{
Condições atuais em termos de perda de biodiversidade \\ em corredores biológicos da Fazenda Agroecológica \\ Uniminuto, Villavicencio, Meta, Colômbia
}

\begin{abstract}
Guillermo González Jiménez ${ }^{1}$ \& Nélvar Choque Ladino²
${ }^{1}$ Administrador de Empresas Agropecuarias, Especialista Producción Agrícola Tropical Sostenible, Estudiante de Maestría en Producción Tropical Sostenible en la Universidad de los Llanos.

${ }^{2}$ Médico Veterinario Zootecnista

${ }^{1,2}$ Corporación Universitaria Minuto de Dios - UNIMINUTO, Semillero de Investigación Agroecosistemas Sostenibles de la Orinoquia Colombiana - ASOC, Villavicencio - Meta, Colombia.
\end{abstract}

19gugonzalez@Uniminuto.edu, ²nelvarchoque@hotmail.com

\section{Resumen}

La Granja Agroecológica Uniminuto, ubicada en el Municipio de Villavicencio, Meta, Colombia, hace parte de la biomicrocuenca del río Guayuriba, lugar donde se realizó un proceso de reconocimiento de condiciones actuales en términos de pérdida de biodiversidad en corredores biológicos, con el propósito de desarrollar propuestas que permitan proteger los ecosistemas y cuidar y conservar las especies nativas de fauna y flora, generando procesos de sensibilización y concientización en las comunidades de la zona. Metodológicamente, a partir del levantamiento de la línea base, se obtuvo información a través de herramientas de recolección primaria: encuestas, registros fotográficos, trabajos de extensión con la comunidad, observación y salidas de campo. Los resultados indican que a pesar del alto nivel de fragmentación, defo- restación, pérdida de conectividad y de coberturas vegetales e intervención humana, aún existe gran biodiversidad de especies (fauna y flora) las cuales sirven como bio-indicadores de dinámicas poblacionales y regeneración natural de ecosistemas. Del orden de los primates, se identificó la especie Callicebus ornatus, localmente aislada en un relicto boscoso, la cual se halla amenazada en estado vulnerable y con una circulación restringida a los corredores objeto de estudio donde ubica sitios específicos de alimentación. Se concluye que existe un alto nivel de intervención humana en los corredores biológicos y relictos boscosos pero es notable la resiliencia de los ecosistemas.

Palabras clave: bioindicadores, conservación, conectividad, fauna, flora 


\section{Abstract}

The Uniminuto agro-ecological farm, located in the city of Villavicencio, Meta, Colombia, is part of the biomicrocuenca of the Río Guayuriba, place where a process of recognition of current conditions was stablished in terms of loss of biodiversity in biological corridors, in order to develop proposals to protect ecosystems and take care and preserve native species of fauna and flora generating processes of sensitization and awareness-raising in communities in the area. Methodologically, the rising of the baseline information was collected through primary collection tools: surveys, photographic records, works of extension with the community, observation, and field trips. The results indicated that despite the high level of fragmentation, deforestation, loss of connectivity and plant cover and human intervention, there is still great biodiversity of species (fauna and flora) which serve as bio-indicators of the population dynamics and natural regeneration of ecosystems. Within the order of the primates, it was identified the specie Callicebus ornatus, locally isolated in a forest relict, which it is threatened in a vulnerable state and with circulation restricted to the corridor, object of study where it were located specific feeding sites. It was concluded that there is a high level of human intervention in forest relicts and biological corridors but the resilience of ecosystems is remarkable.

Key-words: bioindicators, conservation, connectivity, fauna, flora

\section{Resumo}

A granja agroecológica Uniminuto, localizada no município de Villavicencio, Meta, Colômbia, faz parte da bio-microcuenca do rio Guayuriba. Em corredores biológicos desta granja, realizou-se um processo de reconhecimento das condições atuais em termos de perda de biodiversidade, visando desenvolver propostas que permitam proteger os ecossistemas e conservar as espécies nativas de fauna e flora, gerando assim processos de sensibilização e conscientização nas comunidades da área. A partir da linha base, informações foram obtidas por meio de ferramentas primárias de coleta: entrevistas, registros fotográficos, trabalhos de extensão com a comunidade, observação e saídas de campo. Os resultados indicam que apesar do elevado nível de fragmentação, desmatamento, perda de conectividade, coberturas vegetais e intervenção humana, ainda existe grande biodiversidade de espécies (fauna e flora), que servem como bioindicadores da dinâmica populacional e regeneração natural de ecossistemas. Da ordem dos primatas, se identificou a espécie Callicebus ornatus, localmente isolada em um remanente arborizado, a qual está ameaçada, em estado vulnerável e com circulação restringida aos corredores em estudo onde encontra sítios específicos de alimentação. Foi concluído que existe um elevado nível de intervenção humana em corredores biológicos e remanentes de floresta, porém, com destacada resiliência dos ecossistemas.

Palavras-chave: Biomarcadores, armazenamento, conectividade, fauna, flora

\section{Introducción}

El departamento del Meta, Colombia, cuenta con una extensión de $85.635 \mathrm{Km}^{2}$ en los cuales se desarrollan diversos proyectos de tipo agroindustrial cómo son ganadería, cultivos de cereales, palma de aceite, caucho, entre otros, actividades que han generado cambios sustanciales en los ecosistemas en términos de pérdida de biodiversidad, cambios en el uso de la tierra, presión sobre los

recursos naturales, contaminación de fuentes hídricas y fragmentación de hábitat.

La visión de la Corporación Universitaria Minuto de Dios - UNIMINUTO es generar procesos de innovación social incluyente. Esto quiere decir, que se busca apoyar el desarrollo del hombre de una manera integral, donde sea capaz de vivir en 
armonía con su familia, su comunidad y los ecosistemas, generando procesos sostenibles, el cual es el esfuerzo que hace la institución a través de la Granja Agroecológica en Villavicencio, Meta.

Reconocer el entorno de la Granja y entender las dinámicas que allí se generan es el primer paso que se ha dado para direccionar y proyectar sus acciones y esfuerzos. Este predio se ubica a 15 $\mathrm{km}$ de la cabecera municipal, en una zona de vocación y dedicación agropecuaria, con grandes asentamientos humanos y grandes extensiones de ecosistemas naturales como esteros, morichales, lagunas y ríos. Las dinámicas poblacionales y productivas han llevado a generar grandes presiones en la biomicrocuenta del rio Guayuriba, área a la que pertenece la Granja Agroecológica.

Como respuesta a esta situación, el proyecto desarrolla un trabajo de ecología del paisaje de acuerdo con lo descrito por Gurrutxaga, (2008) y Ross,(2006) y tomando el concepto de Cardenal (2002), Mateucci (2014) y García (2012) respecto al área de influencia de la Granja Agroecológica Uniminuto, donde se busca reconocer la importancia de los corredores biológicos como autopistas naturales de desarrollos ecosistémicos de especies nativas de fauna y flora cuyo objetivo es el fortalecimiento de estas estructuras mediante procesos de sensibilización y vinculación activa de las comunidades sobre el cuidado y conservación de los recursos naturales y de promover el establecimiento de sistemas de producción - conservación en los predios vinculados al proyecto con actividades agropecuarias.

\section{Materiales y métodos}

En la Granja ubicada en la vereda Barcelona, en el Municipio de Villavicencio, Meta, Colombia, con coordenadas N4 04.177 W73 35.162 (Figura 1), una altitud promedio de $393 \mathrm{msnm}$, temperatura promedio de $28^{\circ} \mathrm{C}$, topografía plana, precipitaciones anuales entre los $3000 \mathrm{~mm}$ y $4500 \mathrm{~mm}$ por año y una humedad relativa del $70 \%$, se desarrolló la siguiente metodología:

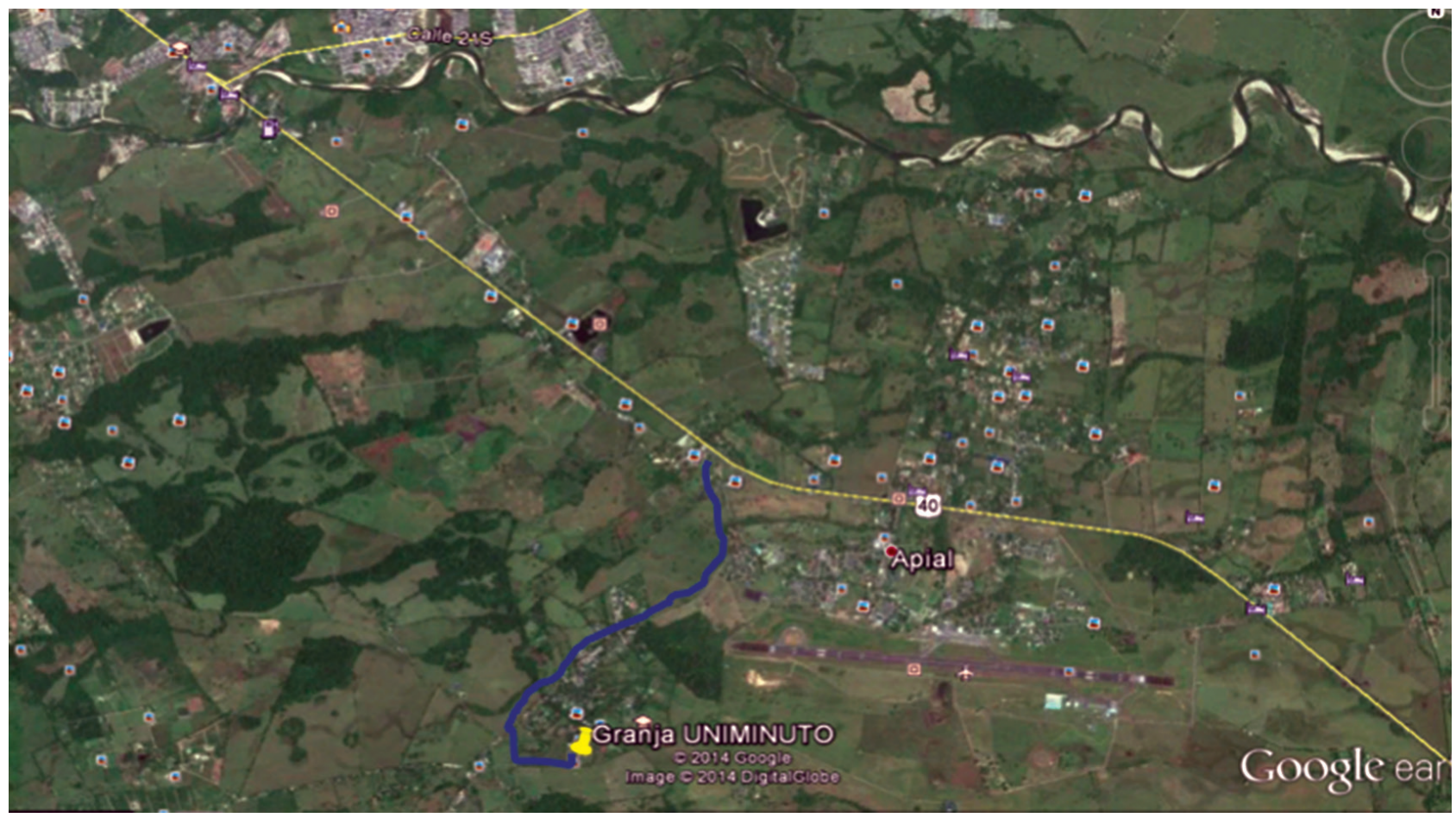

Figura 1. Localización Geográfica Granja Agroecológica Uniminuto 
El área total del estudio es de 1214 ha y un total de $5,8 \mathrm{~km}$ lineales, en los cuales se incluyeron 9 predios, donde se consideró su denominación real y su área en ha, discriminadas así: predio las delicias 360 ha; Loreto: 100 ha; Club gana- dero: 1,5 ha; Hacienda Barcelona: 700 ha; Villa las Flores: 0,625 ha; Granja Agroecológica Uniminuto: 5 ha; Cascarota: 23 ha; Granja Unillanos: 22 ha; El vivero 2 ha; para un total de 1214 ha (ver Tabla 1 y Tabla 2).

Tabla 1. Ubicación georreferenciada del área de estudio.

\begin{tabular}{|c|c|c|}
\hline \multicolumn{3}{|c|}{ Costado Noroccidental } \\
\hline Desde coordenadas & Hasta coordenadas & Localización \\
\hline $4^{\circ} 04 \% 43.97 ” \mathrm{~N}$ & 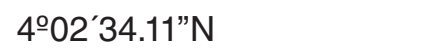 & Parche de bosque de Apiay \\
\hline 733’39.37" O & $73 \div 36^{\prime} 08.48 ” 0$ & \\
\hline Altitud 380 msnm & Altitud 395 msnm & \\
\hline \multicolumn{3}{|c|}{ Costado Sureste Hacienda Barcelona } \\
\hline Desde coordenadas & Hasta coordenadas & Localización \\
\hline $4^{\circ} 02^{\prime} 34.11^{\prime \prime} \mathrm{N}$ & $4^{\circ} 03^{\prime} 05.35^{\prime \prime} \mathrm{N}$ & Extremo sur este \\
\hline $73^{\circ} 36^{\prime} 08.48 ” 0$ & $73^{\circ} 33^{\prime} 25.40^{\prime \prime} \mathrm{O}$ & Hacienda Las Delicias \\
\hline Altitud 395 msnm & Altitud 370 msnm & \\
\hline \multicolumn{3}{|c|}{ Costado Noreste Finca Club Ganadero } \\
\hline Coordenadas inicio & Coordenadas final & Predio \\
\hline $4^{\circ} 03^{\prime} 05.35^{\prime \prime} \mathrm{N}$ & 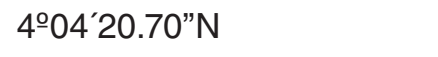 & Granja Agroecológica \\
\hline $73 \cong 33^{\prime 2} 25.40 " \mathrm{O}$ & 733'47.92" O & Uniminuto \\
\hline Altitud 370 msnm & Altitud $371 \mathrm{msnm}$ & \\
\hline
\end{tabular}


Tabla 2. Transectos georreferenciados del área de estudio

\section{Transecto 1}

\begin{tabular}{|c|c|c|}
\hline Coordenadas inicio & Coordenadas final & Predio \\
\hline $4^{\circ} 03^{\prime} 37.57^{\prime \prime} \mathrm{N}$ & 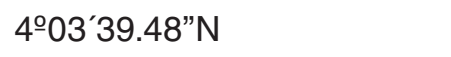 & Hacienda Barcelona \\
\hline 733'45.94" O & $73^{\circ} 34^{\prime} 48.76 " \mathrm{O}$ & \\
\hline Altitud 388 msnm & Altitud 389 msnm & \\
\hline \multicolumn{3}{|c|}{ Transecto 2} \\
\hline Coordenadas inicio & Coordenadas final & Predio \\
\hline $4^{\circ} 04^{\prime} 20.73 ” \mathrm{~N}$ & 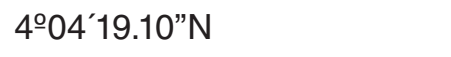 & Granja Agroecológica \\
\hline 733'08.16” O & $73^{\circ} 35^{\prime} 05.26^{\prime \prime} \mathrm{O}$ & Uniminuto \\
\hline Altitud 388 msnm & Altitud 389 msnm & \\
\hline \multicolumn{3}{|c|}{ Transecto 3} \\
\hline Coordenadas inicio & Coordenadas final & Predio \\
\hline $4^{\circ} 04^{\prime} 28.98^{\prime \prime} \mathrm{N}$ & $4^{\circ} 04^{\prime} 26.52^{\prime \prime} \mathrm{N}$ & Finca Club Ganadero \\
\hline $73 \cong 344^{\prime} 44.06 " \mathrm{O}$ & 733ㄴㄴ.83” O & \\
\hline Altitud 387 msnm & Altitud 388 msnm & \\
\hline
\end{tabular}

Nota: los puntos corresponden al punto inicial y punto final de cada transecto.

The Nature Conservancy (McCormick, 2007) ha desarrollado y aplicado una metodología que provee las bases científicas y métodos analíticos al investigador, los cuales permiten definir cómo y dónde conservar y de igual forma, permite medir la efectividad de los procesos que se emprendan. Esta metodología se apoya en cuatro principios a saber; establecimiento de metas y prioridades, desarrollo de estrategias, toma de acciones y medición de resultados.

Para la fase del establecimiento de metas y prioridades, se recolectó la información primaria de los productores, actores ambientales y sociales del área de influencia directa del proyecto y simultáneamente, se realizaron actividades de campo para explorar los componentes de flora y fauna, factores asociados a la conectividad, fragmentación y biodiversidad presentes. Esta fase se desarrolló mediante cuatro etapas:

\section{Aproximación a los índices relativos de flora y fauna.}

Aquí se integró la comunidad en procesos de recolección de información primaria para identificar las especies de flora y fauna presentes en las áreas del proyecto. Para esto, se realizaron visitas guiadas a los predios, donde se socializó el proyecto en términos de objetivos y metodología propuestos. Dicha información se recolectó a través de entrevistas y encuestas previamente diseñadas en las cuales se buscó identificar la presencia de flora y fauna reconocida por los habitantes en 
cada una de las unidades productivas. Adicionalmente se recolectó información relacionada con el área total de los predios explorando las posibles causas de la pérdida de conectividad en los corredores biológicos.

Con esta información se realizó una aproximación a indicadores de abundancia relativa de especies de flora y fauna. Para ello, a partir de fotografías satelitales tomadas del software Google Earth y visitas de campo, se realizó el diseño de transectos en banda, modificados según lo sugerido por Ojasti (2000) Esta actividad se orientó a verificar la información reportada por los habitantes de los predios sobre la presencia de especies de fauna y flora. Adicionalmente, se verificó el estado de los relictos de bosque, parches y corredores biológicos presentes y su relación con biomicrocuenca del rio Guayuriba. Se hizo una clasificación de los ecosistemas presentes según Ruíz (2012) y se realizaron nuevas visitas de campo para verificar su existencia y extensión.

Para la identificación de las especies se emplearon dos métodos: el primero incluyó las encuestas para la recolección de la información, donde se indagó sobre las especies (Ruíz, 2012); La segunda estrategia consistió en ejecutar salidas de campo en las cuales se diseñaron transectos en banda modificados y avistamientos con la técnica de parcelas circulares modificadas, metodología mediante la cual se pudo contar individuos en una extensión, detectados alrededor de un punto central y de esta forma, se estimaron las áreas de las cuales proceden los registros. Esta estrategia ha sido empleada con éxito en avistamientos de aves según lo reporta Ramsey \& Scott (1981) y Roeder et al. (1987), reportados por Ojasti (2000). A partir de la información recolectada con los productores locales sobre el componente vegetal se realizó un registro fotográfico comparativo mediante herbarios digitales, según el citado por Zita (s.f.). Adicionalmente y como una estrategia para empoderamiento con los productores se les presentó el material fotográfico para reconocer las plantas según su denominación común. De este ejercicio se obtuvo el listado de especies vegetales presentes en el área de influencia directa del proyecto.

\section{Sistematización de la información.}

La información recolectada en cada una de las unidades productivas fue sistematizada mediante cuadros de frecuencia y estadística descriptiva; de ellos se ha extraído la lista de especies de flora y fauna y la frecuencia de presentación de especies por predio.

\section{Socialización.}

En el proyecto, el contacto y participación de la comunidad en cada una de las fases fue fundamental. Un primer momento de socialización se llevó a cabo mediante la realización de las entrevistas en las cuales se exploró la percepción de los propietarios y encargados de las unidades productivas respecto de la presencia de especies de flora y fauna así como del desarrollo del proyecto. Para las estrategias de procesos de restauración forestal se previeron jornadas de trabajo comunitario y socialización de objetivos, estrategias, avances y resultados.

\section{Estrategias de recuperación de los corredores.}

Restauración forestal: a partir del análisis de la información recolectada y la clasificación de las coberturas (Ruíz, 2012), se plantea la necesidad de intervenir las áreas mediante varias estrategias de restauración y el restablecimiento de la conectividad en las diferentes unidades productivas. Se establecieron responsabilidades en la consecución y suministro de material vegetal así como la construcción del cronograma de actividades acorde con la disponibilidad de personal.

Se realizaron compromisos para proteger sucesiones ecológicas en áreas denominadas de conservación, en las cuales los productores instalan cercas para evitar el ingreso de ganado o elementos que provoquen la fragmentación (García, 2012), al igual que se evita el uso de guadañas y herbicidas. 
Teniendo en cuenta las metas del Plan de Gobierno Departamental (Gobernación del Meta, s.f.), respecto de los compromisos ambientales sobre cambio climático, se realizaron los contactos y gestión necesaria para obtener el material vegetal para iniciar la ejecución de una de las estrategias propuestas, que tiene que ver con la restauración forestal de corredores, para mejorar y restablecer la conectividad del parche de bosque de la Base Aérea Apiay con el corredor del río Guayuriba. A continuación se presenta la relación de especies forestales (Tabla 3), suministrada por la Secretaria del Medio Ambiente y Recursos Minero Energéticos del Meta.

Tabla 3. Especies forestales suministradas por SMARME.

\begin{tabular}{cclc}
\hline No & Especie & \multicolumn{1}{c}{ Nombre Científico } & Cantidad \\
\hline 1 & Igüa & Pithecellobium guachapele & 150 \\
2 & Yopo & Anadenanthera peregrina & 150 \\
\hline 3 & Ceiba bonga & Ceiba pentandra & 100 \\
\hline 4 & Pan de año & Artocarpus altilis & 100 \\
\hline 5 & Cámbulos & Erythrina poeppigiana & 100 \\
\hline 6 & Guácimo & Guazuma ulmifolia & 150 \\
\hline 7 & Caquín amarillo & No disponible & 100 \\
\hline 8 & Nogal cafetero & Cordia alliodora & 100 \\
\hline
\end{tabular}

Fuente: SMARME: Secretaria del Medio Ambiente y Recursos Minero Energéticos del Meta.

\section{Resultados}

A continuación se presentan las especies forestales reportadas en las unidades productivas (ver Tabla 4), las cuales tienen mayor presencia en los predios de Granja Unillanos, Granja Uniminuto y hacienda Barcelona. Las especies censadas en el proyecto hacen parte integral de los corredores biológicos, por eso no se menciona ningún tipo de clasificación específica, solo se ha tenido en cuenta el servicio ecosistémico que presta, ya sea de aprovisionamiento, regulación, cultural o de soporte (Balvanera, 2012). En la Figura 2 se muestra la distribución de especies forestales por unidad productiva.

Tabla 4. Especies forestales reportadas en las unidades productivas objeto de estudio.

\begin{tabular}{ll}
\hline Especie de flora reportada (NC) & Nombre científico \\
\hline Mango & Mangifera indica \\
Mandarina & Citrus nobilis \\
\hline Limones & Citrus limonum \\
\hline Pinos & Pinus sylvestris \\
\hline Palmas & Flia Arecaceas \\
\hline Pomarroso & Eugenia malaccensis
\end{tabular}


...continuación Tabla 4

\begin{tabular}{|c|c|}
\hline Especie de flora reportada (NC) & Nombre científico \\
\hline Caño fistol & Cassia moschata \\
\hline Mora & Rubus glaucus \\
\hline Guarataro & Mouriri huberi \\
\hline Laurel & Laurus nobilis \\
\hline Yopo & Anaderanthera gigantea \\
\hline Samán & Pithecellobium saman \\
\hline Dormidero & Abies alba \\
\hline Guamo & Inga heteróptera \\
\hline Tuno & Axinaea macrophyla \\
\hline Cucuo & Cuculus canorus \\
\hline Ceiba & Ceiba sp \\
\hline Cedro & Cedrela sp \\
\hline Malagueto & Xilopia aromatica \\
\hline Yarumo guarumo. & Cecropia peltata \\
\hline Gualanday & Jacaranda caucana \\
\hline Flor amarillo & Tabebuia chrysanhta \\
\hline Algarrobo & Ceratonia siliqua \\
\hline Acacia & Acacia $s p$ \\
\hline Bambú & Bambusa vulgaris \\
\hline Casco de vaca & Bauhinia variegata \\
\hline Ficus & Ficus benjamina \\
\hline Igua & Pithecellobium guachapele \\
\hline Botón de oro & Ranunculus bullatus \\
\hline
\end{tabular}




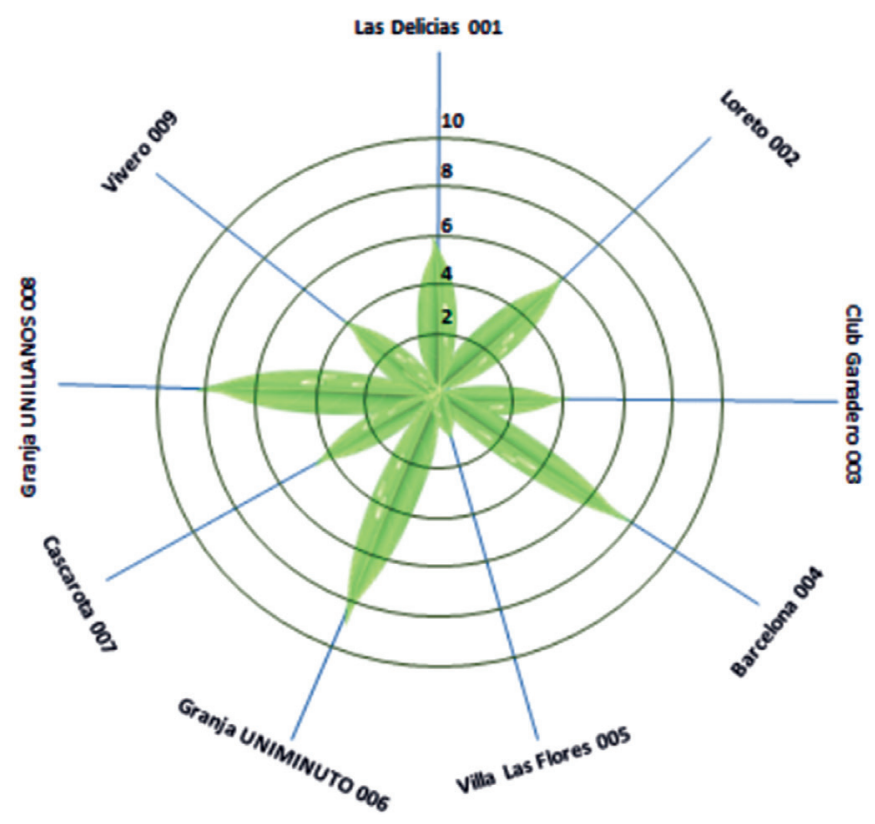

Figura 2. Distribución de especies forestales por unidad productiva objeto de estudio

En las Tablas 5, 6 y 7 se muestran las especies de mamíferos, aves y reptiles encontrados en la zona de estudio. En la Figura 3 se observan las especies de fauna reportadas para las unidades productivas objeto de estudio

Tabla 5. Especies de mamíferos reportadas en las unidades productivas objeto de estudio.

\begin{tabular}{ll}
\hline Especie de mamíferos reportados (NC) & Nombre científico \\
\hline Zorro & Cerdos cyonthous \\
\hline Oso palmero & Mirmecophaga tridactyla \\
Mono araguato & Alouata seniculus \\
\hline Ardillas & Scirius granatencis \\
\hline Cachicamo & Daisypus sp \\
\hline Titi ornamentado, okay & Callicebus ornatus \\
\hline Puerco espino & Histryx pumila \\
\hline Venados & Odocoileus virginianus \\
\hline Oso mielero & Tamandua tridactyla \\
\hline Chucha & Didelphis marsupialis \\
\hline Perro de agua & Lontra longicaudis \\
\hline Perezoso & Choloephus didactylus \\
\hline Erizo & Erinaceus sp \\
\hline
\end{tabular}


Tabla 6. Especies de aves reportadas en las unidades productivas objeto de estudio.

\begin{tabular}{|c|c|}
\hline Nombre Común & Nombre científico \\
\hline Golondrinas & Progenie sp (subis) \\
\hline Pericos & Brotogerisjugularis \\
\hline Ciriguelos & Crotophaga (ani o major) \\
\hline Azulejos & Passerinacyanea \\
\hline Arrendajos & Casicus cela \\
\hline Canarios & Serinus canaria \\
\hline Colibrís & Familia Trochilidade \\
\hline Garza ganadera & Bubulcus ibis \\
\hline Guacharacas & Ortalis ruficauda \\
\hline Pechi amarillo & Tolmomyias flaviventris \\
\hline Alcaravanes & Burhinus bistriatus \\
\hline Loros carisucio & Aratinga pertinax \\
\hline Chorolas & No identificadas \\
\hline Pechiblancos & No identificadas \\
\hline Carpinteros & Melanerpes sp; Dryocopos sp \\
\hline Tucán & Ramphastus tucanus \\
\hline Abuelitas & Genero Columbidos \\
\hline Chulos & Coragyps atratus; Cathartes aura \\
\hline Mirla embarradora & Turdus ignobilis \\
\hline Chilaco & Aramides cajenea \\
\hline Aguilas & No identifiadas \\
\hline Pímparos & Pitangus sulfuratus \\
\hline Pollo de monte & No identificado \\
\hline Caica & Vanellus chilencis \\
\hline Loro real & Amazona ocrocephala \\
\hline
\end{tabular}


Tabla 7. Especies de reptiles reportadas en las unidades productivas objeto de estudio

\begin{tabular}{ll}
\hline Nombre común & Nombre científico \\
\hline Serpiente cuatro narices & Bothrops $s p$ \\
\hline Serpiente coral & Micrurus sp \\
\hline Iguanas & Iguana iguana \\
\hline Lagartijas & Anolis sp \\
\hline Garipiare & Tupinambis teguixin \\
\hline Cazadora verde & Chironius sp \\
\hline Güios & Boa cosntrictor c. \\
Babilla & Caiman crocodilus c. \\
Cachirre & Paleosuchus sp. \\
\hline
\end{tabular}

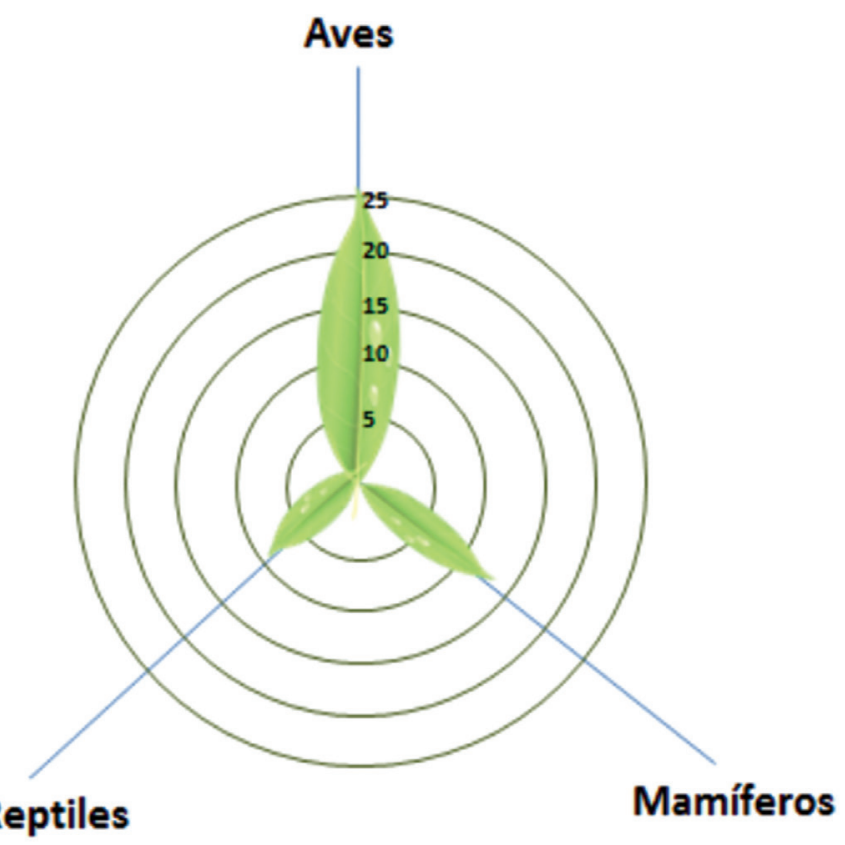

Figura 3. Especies de fauna reportadas para las unidades productivas objeto de estudio.

Para las especies de fauna identificadas se registró información relacionada con el comportamiento, desplazamiento y presencia de especies, particularmente mamíferos, aves y su relación con el componente forestal. Se determinaron zonas de alimentación para la especie Callicebus ornatus, (Mico okay) (Bueno, 2010) el cual en compañía del grupo social se desplaza a través de las instalaciones de la Universidad de los Llanos para alimentarse en la zona de reserva de la Granja Uniminuto. Esto evidencia un alto nivel de adaptación de esta especie, situación que es posible determinar por las distancias de fuga con respecto al observador, la cual en algunos casos no sobrepasa los $12 \mathrm{~m}$.

Respecto a la presencia de aves, se constituye en el grupo taxonómico con mayores registros 
indistintamente de las unidades productivas. Usan principalmente flores, frutos y semillas para su alimentación, así como troncos para su reproducción. Estos datos permiten afirmar que si bien existe un alto nivel de intervención en los relictos de bosque y corredores biológicos, es notable la resiliencia de los ecosistemas de acuerdo a lo expresado por Hernández (2009) y un alto grado de adaptación de las especies a los cada vez más frecuentes cambios en la cobertura vegetal, la cual está asociada a otros factores como cambio en el uso del suelo, construcción de viviendas y obras de infraestructura como vías.

Las especies forestales prestan el soporte alimentario y sustrato para la movilidad y la reproducción a la mayoría de especies animales, existiendo un alto nivel de dependencia de la supervivencia de las especies de fauna. Con el sustrato forestal de acuerdo con lo expresado por García (2012), aspecto integrado al manejo comunitario el cual se basa en la adquisición y comunicación de la información mediante estrategias que logran hacer que la población directamente afectada implemente el manejo de las poblaciones de fauna a través de su conservación. Paralelamente, la investigación y la promoción agroforestal con énfasis en los servicios ecosistémicos y recursos agroforestales, suministra información sobre el mejoramiento de hábitats de fauna silvestre y la efectividad de las estrategias de manejo. (Bodmer \& Puertas, 2000).

Las especies forestales reportadas toman gran importancia pues hacen parte de las intrincadas redes tróficas, de relaciones simbióticas, parasitarias y son sustrato para el desarrollo de la trama de vida local; de ahí la importancia de conservarlas e incluirlas en el fortalecimiento de los corredores en las fincas de los productores objeto de intervención. (Noss, 2006).

Como producto de la fase establecimiento de metas y prioridades se resalta el diseño del trazado de los corredores biológicos mediante acuerdo de voluntades con los productores de los predios, sobre los cuales se desarrollan las iniciativas de restauración forestal, lo cual permitirá mejorar la movilidad de las especies de fauna silvestre, mejorar el microclima local, aumentar la oferta de los servicios ecosistémicos de la zona, entre otros.

\section{Discusión}

La información aquí presentada respecto de los hallazgos en los temas de flora y fauna y el estado de conservación o afectación de los ecosistemas locales, permite afirmar según lo descrito por McCormick, (2007), que la integración de factores determinantes en la intervención de los ecosistemas exige una alta responsabilidad de la participación comunitaria como una apropiación del modelo metodológico en el éxito de programas de conservación, el cual debe ser constante y retroalimentado de manera permanente.

Si bien se concluye que existe un alto nivel de intervención humana en los corredores biológicos y relictos boscosos, es notable la resiliencia de los ecosistemas en concordancia con lo propuesto por Hernández (2009) y un alto grado de adaptación de las especies a los cada vez más frecuentes cambios en la cobertura vegetal, manifiestos en la pérdida de conectividad observados en las salidas de campo, donde también es notable la disminución de las distancias de fuga (distancia entre el observador y el espécimen) de especies de primates, caso puntual callicebus ornatus, especie cuyos individuos hacen uso de cuerdas y techos para su desplazamiento a las zonas de alimentación ubicadas en la Granja Agroecológica Uniminuto, mostrando un alto nivel de adaptabilidad, lo cual pude ser positivo para la supervivencia de la especie.

Respecto del establecimiento de metas y prioridades. McCormick (2007) en el fortalecimiento de los corredores biológicos, se comprueba que es necesaria la directa participación de los productores de las unidades productivas intervenidas, dado que ellos son los primeros beneficiados con el uso de los servicios ecosistémicos (Balvanera, 2012), pero 
también en el caso de los fracasos son ellos quienes deben asumirlo. Es importante resaltar que aun existiendo mucha información sobre la conservación y particularmente sobre los efectos ambientales producto del cambio climático, del régimen de lluvias y de especies tanto de fauna como de flora en vía de extinción, aún existe resistencia por parte de algunos productores para adoptar estas estrategias de conservación y restauración ecológica, además se identifican ciertos mitos respecto de los sistemas de conservación, por parte de los productores los cuales deben ser tenidos en cuenta en los proceso de conciliación de los acuerdos para el establecimiento de las metas.

Como resultado de la implementación de estrategias, se tiene la adopción de la especie de primate Callicebus ornatus conocido como titi ornamentado u okay, como especie bandera del semillero de investigación ASOC, Agroecosistemas sostenibles de la Orinoquia, el cual será promovido a través de chalecos y cachuchas las cuales llevarán este distintivo como una forma de visibilizar la especie y dar a conocer a la comunidad la importancia de la conservación de este recurso en la sostenibilidad de los servicios ecosistémicos. También se prevé la socialización y masificación de esta estrategia con los actores de la gestión en el área de influencia directa del proyecto.

\section{Conclusiones}

Existe un alto nivel de intervención de las comunidades en los relictos, parches de bosques y corredores biológicos, lo cual se evidencia por la pérdida de conectividad y el encerramiento de algunas especies, como es el caso de los primates. Adicionalmente es notable la construcción de obras de infraestructura que afectan de manera colateral los recursos y los servicios ecosistémicos.

Existe acuerdo en la adopción de la metodología de The Nature Conservation, para desarrollar la intervención en la comunidad y los ecosistemas con propósitos de conservación; esta metodología en la práctica ha demostrado ser una herramienta adecuada y pertinente para el establecimiento de metas y prioridades.

Es posible a partir del listado de especies de flora y fauna (aves, mamíferos y reptiles) determinar que a pesar del alto grado de intervención, algunas de estas especies exhiben un gran nivel de adaptación a las nuevas y particularmente cambiantes condiciones.

Finalmente, se decide iniciar el registro de la Granja Agroecológica Uniminuto como Reserva Natural de la Sociedad Civil "Rafael García Herreros". Este proceso empieza a partir del resultado de este proyecto ante Parques nacionales y se adoptará como estrategia de conservación de áreas propias y vecinas. Con esta certificación se definirán exactamente las zonas de conservación, de amortiguación y manejo especial, de agroecosistemas, uso intensivo e infraestructura.

\section{Agradecimientos}

Los autores expresan su agradecimiento a la Corporación Universitaria Minuto de Dios por facilitar los medios para hacer investigación especialmente en el área ambiental; a la Gerencia Ambiental y Minero Energética, dependencia de la Gobernación del Meta por el suministro del material vegetal.

\section{Literatura citada}

1. Balvanera, P. (2012). Asociación Española de Ecología Terrestre. AEET Los servicios ecosistémicos que ofrecen los bosques tropicales. Recuperado de http://www. revistaecosistemas.net/articulo.asp? $\mathrm{ld}=709$.

2. Bodmer, R. \& P. Puertas. (2000). Community Based CoManagement of Wildlife in the Peruvian Amazon, en: J. Robinson and L. Bennet (eds.): Hunting of Tropical Wildlife, University of Chicago Press. Recuperado de http://sisbib.unmsm.edu.pe/bvrevistas/biologia/v07_n2/ biblio_impor_mane.htm

3. Bueno. M. \&, Defler T. (2010). Aportes citogenéticos en el esclarecimiento de la taxonomía del género Callicebus. Orinoquia 14 sup (1):139-152. 
4. Cardenal, L (2002). Proyecto para la Consolidación del Corredor Biológico Mesoamericano. El Corredor Biológico Mesoamericano: una plataforma para el desarrollo sostenible regional/ Proyecto para la consolidación del Corredor Biológico Mesoamericano. 1a ed. Managua: Proyecto Corredor Biológico Mesoamericano, 24p.

5. Hernandez. E. L. \& Chacón. J. P. (2013). Nuevo registro de Myrmecophagatridactylapara el departamento de córdoba, Colombia con anotaciones. Sobre comportamiento agonístico interespecífico Grupo de Biodiversidad Unicordoba, Facultad de Ciencias Básicas. Departamento de Biología. Universidad de Córdoba.

6. García, J. R. (2012). Amazonia Posible y Sostenible. Corredores Biológicos en la Amazonia Colombiana: Estado actual, amenazas y sostenibilidad. Bogotá, Colombia. CEPAL.

7. Gobernación del Meta (s.f.). Plan de Desarrollo Juntos Construyendo Sueños y Realidades 2012 - 2015. Recuperado de: http://webcache.googleusercontent.com/ search?q=cache:cMikP0nWUBkJ:www.meta.gov.co/ es/plan-de-desarrollo/+\&cd=1\&hl=es\&ct=clnk

8. Gurrutxaga. M. \& Lozano V .P. (2008). Ecología del Paisaje. Un marco para el estudio integrado de la dinámica territorial y su incidencia en la vida silvestre. P. 524.

9. Hernández, B. M. (2009). La resiliencia de los ecosistemas, clave para el desarrollo sostenible. Revista CEGESTI, Volumen. 9

10. Matteucci, S.D. (2014). El análisis regional desde la ecología. Sistemas Ambientales Complejos: herramientas de análisis espacial. Colección CEA № 21; EUDEBA, Buenos Aires; pp 117-150.
11. McCormikc, S. (2007). The Nature Conservancy, Diseño para la Conservación. Un marco estratégico para el desarrollo de la Misión.

12. Noss, R. F. \& Daly, K. M. (2006): “Incorporating connectivity into broad-scale conservation planning", en Crooks, K. y Sanjayan, M. (eds.), Connectivity Conservation, Cambridge. University Press, Cambridge, pp. 587-619.

13. Ojasti J. \& Dallmeier, F. (ed.). (2000). Manejo de Fauna Silvestre Neotropical. SI/MAB. Series \# 5. Smithsonian Institution /MAB Biodiversity Program, Washington. D.C.

14. Ruiz O., C., Cardona, H. \& Duque, J. L. (2012). Corredores biológicos una estrategia de recuperación en paisajes altamente fragmentados. Estudio de caso Microcuenca La Bolsa, municipio de Marinilla. Gestión y Ambiente, vol. 15, núm. 1, febrero-mayo, 2012, pp. 7-18

15. Zita, G. (s.f.). Metodología para el envío y preservación de plantas. Facultad de Estudios Superiores Cuautitlán. Universidad Autónoma de México. Recuperado de: http://www.agricolaunam.org.mx/malezas/Zita\% 20Preservacion\%20de\%20plantas.pdf

\section{Conflicto de Intereses}

Los autores declaran no tener ningún conflicto de intereses 